\section{Bem-estar psicológico entre travestis e mulheres transexuais no Estado de São Paulo, Brasil}

\author{
Psychological well-being among transvestites and \\ trans women in the state of São Paulo, Brazil
}

\section{Bienestar psicológico entre travestis y mujeres transexuales en el estado de São Paulo, Brasil}

\section{Resumo}

O objetivo do trabalho foi estimar fatores associados ao bem-estar psicológico de travestis e mulheres transexuais. Estudo transversal com 602 travestis e pessoas transexuais em sete municípios do Estado de São Paulo, Brasil entre 2014 e 2015. Foi realizada seleção amostral com abordagem consecutiva e técnica bola de neve. A variável dependente foi bem-estar psicológico (WHOQOL-BREF) e as independentes foram: características sociodemográficas, modificações corporais, condições de saúde, violência e encarceramento. A análise de variância múltipla foi usada para identificar os fatores associados. A maioria tinha cor da pele preta ou parda e entre 25 e 39 anos de idade, até o Ensino Médio completo, renda individual de até dois salários mínimos e trabalhava, sendo 42,3\% profissionais do sexo. Cerca de um quarto já foi presa. Em torno de um quarto fazia tratamento para HIV. O escore médio observado foi de 63,2 (IC95\%: 61,8-64,6). Na análise múltipla, estiveram associados ao menor bem-estar psicológico: não ter endereço fixo, ter menor escolaridade, estar insatisfeita com as relações pessoais, suporte de amigos ou procedimentos transexualizadores realizados e ter sofrido violência verbal ou sexual. Enquanto piores condições de vida e de exposição à violência prejudicam o bem-estar psicológico de travestis e mulheres transexuais, a possibilidade de realizar transformações corporais desejadas e o respeito ao nome social interferem positivamente na avaliação que fazem de suas vidas.

Travestismo; Pessoas Transgênero; Saúde Mental
Eliana Miura Zucchi 1

Claudia Renata dos Santos Barros 1

Bruna Robba Lara Redoschi 2

Luiz Fabio Alves de Deus 3

Maria Amélia de Sousa Mascena Veras 4

doi: 10.1590/0102-311X00064618

\section{Correspondência}

E. M. Zucchi

Programa de Pós-graduação em Saúde Coletiva, Universidade

Católica de Santos.

Av. Conselheiro Nébias 300, Santos, SP 11015-002, Brasil.

eliana.zucchi@uol.com.br

1 Programa de Pós-graduação em Saúde Coletiva, Universidade Católica de Santos, Santos, Brasil.

2 Programa de Pós-graduação em Saúde Pública, Universidade de São Paulo, São Paulo, Brasil.

3 Programa de Pós-graduação em Psicologia Social e

Institucional, Universidade Federal do Rio Grande do Sul, Porto Alegre, Brasil.

4 Faculdade de Ciências Médicas da Santa Casa de São Paulo, São Paulo, Brasil. 


\section{Introdução}

Pessoas transexuais, definidas como aquelas cujo sexo designado ao nascimento difere de sua expressão ou identidade de gênero atual, apresentam altos indicadores de iniquidades em saúde, caracterizadas como multifacetadas e resultantes de processos de marginalização socioeconômica, estigma e discriminação 1 .

Paralelamente à construção de agendas globais 2 e de políticas e programas locais em diversos setores 3,4 , a literatura científica sobre saúde da "população transgênero" - termo adotado nas publicações de língua inglesa desde a década de 1990 como um termo guarda-chuva que engloba um amplo espectro de práticas e identidades de gênero variantes, ou seja, em desacordo com o sexo biológico, embora no Brasil não seja universalmente utilizado 5 - tem crescido substancialmente nos últimos anos, sendo a saúde mental um campo que concentra evidências consistentes de que pessoas travestis e transexuais são desproporcionalmente afetadas por problemas neste domínio ${ }^{1}$. No Brasil, a categoria identitária "travesti” aparece na literatura em torno da década de 1970 para designar pessoas que tiveram sexo masculino atribuído ao nascimento, mas que se identificam com o gênero feminino. A categoria "transexual" seria historicamente mais recente, emergindo entre o final dos anos 1990 e o início dos anos 2000 6. As categorias "travesti” e "transexual” são descritas na literatura científica como termos atravessados e carregados de tensões políticas e identitárias e limites não tão claros que extrapolam a finalidade deste estudo, mas que podem ser observadas nos trabalhos de Carvalho \& Carrara 6 e Barbosa ${ }^{7}$. Consideramos neste trabalho a distinção das categorias em respeito às identidades autoatribuídas pelas participantes e também em razão de distinções apontadas que poderiam implicar diferenças nas condições de saúde dessas pessoas. Altas prevalências de transtorno depressivo ou de sintomatologia depressiva 8, ideação suicida 9,10 e uso abusivo de drogas 11 são agravos frequentemente documentados e, em geral, seus determinantes expressam condições de desigualdade socioeconômica (por exemplo, baixa escolaridade, baixa renda, desemprego/subemprego, sexo comercial) 12,13, aspectos psicossociais (tais como heterossexismo internalizado e percepção geral de estresse 8) e experiências de violência e discriminação 14.

No campo da saúde mental, o construto bem-estar psicológico é particularmente valioso para acessar uma vasta gama de percepções e sensações com base na autoavaliação do indivíduo sobre diversos domínios da vida. Embora haja diferentes abordagens no exame desse construto - sendo as principais: avaliação da vida, bem-estar hedônico e bem-estar eudemônico 15 -, suas características centrais incluem a autoaceitação, relacionamentos positivos com outras pessoas, autonomia sobre suas experiências com critérios próprios, domínio sobre o ambiente, propósito na vida e crescimento pessoal 16. Seus principais correlatos psicossociais incluem: autoestima e moral, satisfação de vida e afetos positivos, coping, geratividade, memórias integrativas e intrínsecas, generosidade, habilidades sociais, inteligência emocional, valores intrínsecos, resiliência e metas de crescimento 16. O bem-estar psicológico apresenta convergência com transtornos mentais 17,18, indicadores de bem-estar, qualidade de vida, marcadores biológicos (por exemplo, neuroendócrinos, imunológicos e cardiovasculares) e outros construtos que expressam dimensões positivas da saúde mental 16. Dessa forma, tendo em vista as características específicas desse construto, parte expressiva da literatura sobre bem-estar psicológico o analisa em conjunto com, por exemplo, dimensões relativas a suporte social 19, violência e discriminação 20 , depressão 20 , entre outros. De forma consistente com a literatura sobre os outros indicadores de saúde, quando comparadas a indivíduos cisgênero, pessoas transgênero apresentam pior bem-estar psicológico ${ }^{19}$. Ainda, comparativamente a homens transgênero, pessoas de identidade não binária e indivíduos cisgênero homossexuais e bissexuais, as mulheres transgênero apresentam os piores indicadores de bem-estar psicológico 21, indicando a importância de compreender as especificidades de suas experiências psicossociais e contextos de vida.

As experiências de estigmatização, entendida como processos socialmente construídos de desvalorização de identidades que materializam relações de poder 22 e de discriminação (efetivação do estigma que constitui violação de direitos) 23 têm efeito reconhecido na manifestação de sintomatologias indicativas de sofrimento mental intenso entre pessoas transexuais 1,14,24,25,26,27, e vêm ganhando maior especificidade na identificação das formas e contextos em que ocorrem. Ao analisar o plano estrutural, a inexistência de políticas públicas protetivas repercute em piores indicadores de saúde mental e qualidade de vida. Esse cenário pode ser observado, por exemplo, na pior qualidade de vida 
de mulheres transexuais que sofreram discriminação sistemática por agentes da lei em Shenyang, na China 28, assim como em maiores prevalências de transtornos de humor e de violência autoinflingida entre veteranos transexuais residentes em estados que não tinham leis antidiscriminatórias nos Estados Unidos 29. No plano cultural e simbólico, o racismo também incide de forma mais grave na saúde mental de mulheres transgênero negras, comparativamente a mulheres brancas 30,31. Outra situação contextual geralmente associada à violência é a experiência de encarceramento. Entre mulheres transexuais e travestis 32 o encarceramento exibe contornos particulares que incluem o não reconhecimento da transgeneridade e alocação em celas/alas com base no sexo biológico 23, súbita interrupção do processo transexualizador 33 , estupros e espancamentos, constituindo uma punição arbitrária 33.

No contexto brasileiro, estudos com a população de pessoas transexuais também tiveram expressivo aumento na última década, ainda que majoritariamente usando metodologias qualitativas. Parte significativa dessas investigações científicas se concentra nas experiências dessa população com o mercado do sexo, HIV/aids e mudanças corporais 34. Embora as investigações sobre discriminação e violência contra pessoas transexuais ainda sejam incipientes, os dados disponíveis, frequentemente obtidos com a participação de organizações não governamentais, apresentam uma realidade alarmante, caracterizando uma violência que acomete e atravessa a trajetória de vida das travestis e mulheres transexuais em todos os contextos e situações sociais no Brasil. A violência física figura entre os tipos mais comuns de agressão contra travestis e mulheres transexuais, sendo frequente que seja cometida de maneira cruel e desumana 35,36,37. De acordo com observatório internacional que monitora homicídios de pessoas transexuais em 65 países 38 , o Brasil ocupa a primeira posição no ranking de assassinatos de travestis e mulheres transexuais. Altas prevalências de violência verbal e física são descritas em diversos contextos sociais e, frequentemente, resultam na saída precoce de casa motivada pela não aceitação da identidade de gênero por parte da família, assim como em expulsão da escola, bem como na ausência de busca por serviços de saúde em razão da hostilidade vivida ou pressentida 37,39,40. Em resposta a esse contexto, nos últimos anos alguns municípios e estados brasileiros têm criado dispositivos legais que visam a proteger e coibir a violência contra a população de lésbicas, gays, travestis e transexuais (LGBTT), bem como têm sido criadas delegacias e defensorias públicas que prestam auxílio em casos de discriminação e violência transfóbica. Contudo, tais medidas ainda são localizadas e incipientes 41 .

O objetivo do trabalho foi estimar fatores associados ao bem-estar psicológico de travestis e mulheres transexuais. Procuramos explorar se as diversas formas de violência e discriminação que travestis e mulheres transexuais vivem no cotidiano, bem como condições de desigualdade sociais, afetam negativamente o seu bem-estar psicológico.

\section{Métodos}

Estudo transversal proveniente do Projeto Muriel: Vulnerabilidades, Demandas de Saúde e Acesso a Serviços da População de Travestis e Transexuais do Estado de São Paulo, realizado em sete municípios paulistas (São Paulo, Campinas, São Bernardo do Campo, Santo André, Santos, São José do Rio Preto e Piracicaba). A coleta de dados ocorreu entre 2014 e 2015.

A seleção amostral combinou a abordagem consecutiva com a técnica bola de neve, que tem por base as redes de relações, constituindo uma amostra não probabilística.

Para estabelecer o número de entrevistas em cada município, foi realizado no ano anterior à coleta de dados um levantamento do número total de pessoas matriculadas ou assistidas por serviços de saúde ou de assistência social. Com base nos dados obtidos nesse levantamento e estimando uma perda de $30 \%$ dessa clientela, um total de 700 pessoas seriam potencialmente atingíveis. A distribuição do número de pessoas a ser entrevistadas em cada município foi proporcional ao tamanho da clientela de pessoas travestis e transexuais nos serviços antes mencionados.

Essa abordagem amostral foi utilizada em razão da não disponibilidade de fontes de caráter censitário sobre essa população, caracterizada como de difícil acesso para os estudos epidemiológicos. Foram adotados os seguintes critérios de inclusão: ter 16 anos ou mais na ocasião da entrevista; identificar-se como travesti, transexual, transgênero; e residir no Estado de São Paulo há pelo menos seis meses. 
A amostra total do Projeto Muriel foi de 673 pessoas que se autoidentificaram como travestis, mulher ou homem transexual e transmulheres e transhomens. Neste estudo, utilizamos as identidades travesti e mulher transexual ou transgênero, o que totalizou uma amostra de 602 entrevistas.

A variável dependente foi o bem-estar psicológico estimado baseando-se nos resultados obtidos para o domínio psicológico do WHOQOL-BREF (World Health Organization Quality of Life). Esse escore assume o valor máximo de 100 e mínimo de zero. As variáveis independentes foram: condições sociodemográficas: situação de moradia, categorizada em "sem endereço fixo" e "tem endereço fixo"; anos de escolaridade, com corte em oito anos de estudos; renda mensal individual categorizada por salários mínimos, com salário base de R\$ 788,00; cor da pele segundo o Instituto Brasileiro de Geografia e Estatística (IBGE) e reagrupada em "branca, amarela e indígena”, "preta e parda”; trabalho atual "não" (inclui pessoas que se consideravam desempregadas mas tinham o trabalho sexual como fonte de renda), "sim, como profissional do sexo" e "sim, outras atividades"; ter alguma religião; e ter parceiro fixo no momento da pesquisa. Insatisfação com as relações pessoais e suporte de amigos ("não está insatisfeita" e "está insatisfeita”); relação com a família ("nenhuma”, "conflituosa ou ruim", "aceitável ou boa”); insatisfação média com procedimentos transexualizadores (hormônios, silicone industrial, próteses de silicone e outros procedimentos cirúrgicos), categorizada em "não está insatisfeita", "pouco satisfeita ou insatisfeita” e "não fez nenhum procedimento"; mudança de nome em pelo menos um documento ("não: ainda não tentou, tentou e não conseguiu, não tem documento ou ainda está tentando", "não pretende mudar em nenhum documento" e "sim, tentou e conseguiu”); números de locais em que é tratada pelo nome social; violência categorizada em "não sofreu nenhuma”, "agressão verbal/assédio moral/constrangimento/humilhação (exclusiva)", "física”, "sexual”, "chantagem/ extorsão ou violência policial” (as três últimas categorias da variável de violência não são exclusivas); já ficou presa (por mais de 24 horas); e tratamento para HIV: "sim” ou "não" (autodeclarado).

\section{Análise estatística}

A variável dependente (bem-estar psicológico) foi descrita em médias e desvios padrão. As variáveis independentes foram descritas por meio de frequências absolutas e relativas. Os testes de qui-quadrado de Pearson e exato de Fisher foram usados para testar as variáveis qualitativas.

Os fatores associados ao bem-estar psicológico foram estimados por meio do modelo de análise de covariância bivariada e múltipla. As variáveis que apresentaram $\mathrm{p}<0,20$ na análise bivariada foram selecionadas para o modelo múltiplo. No modelo múltiplo final foram mantidas as variáveis estatisticamente significativas e aquelas que ajustaram as demais variáveis em pelo menos $10 \%$. O nível de significância adotado foi de $5 \%$.

\section{Aspectos éticos}

O Projeto Muriel foi aprovado pelos Comitês de Ética em Pesquisa do Centro de Referência e Treinamento em DST/AIDS-SP (CRT DST/AIDS-SP) (registro CAAE: 14277413.1.0000.5375) e da Secretaria Municipal de Saúde de São Paulo (registro CAAE: 14277413.1.0000.53750) e está em consonância com as diretrizes para pesquisas com seres humanos no Brasil. Todas as participantes assinaram um Termo de Consentimento Livre e Esclarecido (TCLE) antes da condução das entrevistas.

Ainda sobre os aspectos éticos, a necessidade de incluir pessoas a partir de 16 anos foi discutida com os representantes de serviços de saúde, da sociedade civil organizada, ativistas do movimento LGBTT e pesquisadores que atuam com estas populações. Esse procedimento foi feito utilizando-se o conceito de menor responsável, dispositivo que serve para garantir o acesso ao teste anti-HIV em menores de idade, uma vez que o consentimento dos responsáveis pode acarretar problemas e afastar esta população do acesso à testagem ${ }^{42}$. Assim, dado o contexto de extrema marginalização e exclusão que marca a trajetória de vida de travestis e transexuais, sendo o rompimento do vínculo familiar frequente e, comumente, ocorrendo a partir do momento em que o processo de transição de gênero inicia, em muitos casos nos primeiros anos da adolescência 43 , optou-se pela inclusão de participantes a partir dos 16 anos de idade. 


\section{Resultados}

A amostra final foi de 602 mulheres que se autoidentificaram como travestis, transexuais e transgênero. A maioria tinha endereço fixo, oito anos ou mais de estudos, era preta ou parda, tinha alguma religião, não tinha parceiro fixo, tinha renda mensal de até dois ou mais de dois salários mínimos e relatou ter algum trabalho no momento da pesquisa, das quais $42 \%$ referiram ser profissionais do sexo. No que diz respeito às relações, a maioria relatou satisfação em suas relações pessoais, com o suporte de amigos e relação aceitável ou boa com a família (Tabela 1).

\section{Tabela 1}

Descrição da amostra de travestis, mulheres transexuais ou transgênero. São Paulo, Brasil, 2015.

\begin{tabular}{|c|c|c|}
\hline & \multicolumn{2}{|c|}{$\begin{array}{c}\text { Total } \\
(n=602)\end{array}$} \\
\hline & $\mathbf{n}$ & $\%$ \\
\hline WHOQOL-BREF (domínio psicológico) [média; DP] & 63,2 & 17,7 \\
\hline \multicolumn{3}{|l|}{ Situação de moradia } \\
\hline Tem endereço fixo & 490 & 82,1 \\
\hline Sem endereço fixo & 107 & 17,9 \\
\hline \multicolumn{3}{|l|}{ Anos de escolaridade (anos) } \\
\hline$\geq 8$ & 375 & 62,3 \\
\hline$<8$ & 227 & 37,7 \\
\hline \multicolumn{3}{|l|}{ Renda (salários mínimos) } \\
\hline Até meio & 103 & 17,1 \\
\hline Até 1 & 85 & 14,1 \\
\hline Até 2 & 184 & 30,6 \\
\hline Mais de 2 & 230 & 38,2 \\
\hline \multicolumn{3}{|l|}{ Cor da pele } \\
\hline Branca, amarela, indígena & 239 & 39,8 \\
\hline Preta ou parda & 361 & 60,2 \\
\hline \multicolumn{3}{|l|}{ Trabalha } \\
\hline Não & 145 & 24,3 \\
\hline Sim, profissional do sexo & 253 & 42,3 \\
\hline Sim, outras atividades & 200 & 33,4 \\
\hline \multicolumn{3}{|l|}{ Está insatisfeita com as relações pessoais } \\
\hline Não & 490 & 81,7 \\
\hline Sim & 110 & 18,3 \\
\hline \multicolumn{3}{|l|}{ Está insatisfeita com o suporte de amigos } \\
\hline Não & 453 & 75,5 \\
\hline Sim & 147 & 24,5 \\
\hline \multicolumn{3}{|l|}{ Relação com a família } \\
\hline Nenhuma & 55 & 9,1 \\
\hline Conflituosa/Muito ruim & 106 & 17,6 \\
\hline Aceitável/Boa & 441 & 73,3 \\
\hline \multicolumn{3}{|l|}{ Tem alguma religião } \\
\hline Não & 170 & 28,2 \\
\hline Sim & 432 & 71,8 \\
\hline \multicolumn{3}{|l|}{ Tem parceiro fixo } \\
\hline Sim & 195 & 32,5 \\
\hline Não & 405 & 67,5 \\
\hline
\end{tabular}




\begin{tabular}{|c|c|c|}
\hline & \multicolumn{2}{|c|}{$\begin{array}{c}\text { Total } \\
(n=602)\end{array}$} \\
\hline & $\mathbf{n}$ & $\%$ \\
\hline \multicolumn{3}{|l|}{ Está insatisfeita com os procedimentos realizados } \\
\hline Não & 370 & 61,6 \\
\hline Sim (pouco satisfeita/insatisfeita) & 174 & 29,0 \\
\hline Não fez nenhum procedimento & 57 & 9,5 \\
\hline \multicolumn{3}{|l|}{ Tentou e conseguiu mudar o nome em pelo menos um documento } \\
\hline Não (ainda não tentou/tentou e não conseguiu/não tem documento/está tentando) & 539 & 89,7 \\
\hline Não pretende mudar em nenhum dos documentos & 10 & 1,7 \\
\hline Sim & 52 & 8,7 \\
\hline \multicolumn{3}{|l|}{ Número de locais em que é tratada pelo nome social } \\
\hline Até 4 & 177 & 29,4 \\
\hline $4-6$ & 222 & 36,9 \\
\hline 7 ou mais & 203 & 33,7 \\
\hline \multicolumn{3}{|l|}{ Violência } \\
\hline Não sofreu violência & 36 & 6,0 \\
\hline Agressão verbal/Assédio moral/Constrangimento/Humilhação & 115 & 19,1 \\
\hline Agressão física & 73 & 12,1 \\
\hline Violência sexual & 37 & 6,2 \\
\hline Chantagem/Extorsão ou violência policial & 341 & 56,6 \\
\hline \multicolumn{3}{|l|}{ Já foi presa } \\
\hline Não & 438 & 77,8 \\
\hline Sim & 125 & 22,2 \\
\hline \multicolumn{3}{|l|}{ Faz tratamento para HIV } \\
\hline Não & 431 & 71,6 \\
\hline Sim & 171 & 28,4 \\
\hline
\end{tabular}

DP: desvio padrão; WHOQOL-BREF: World Health Organization Quality of Life.

Ainda, como apresentado na Tabela 1, 62\% estavam satisfeitas e 29\% pouco satisfeitas ou insatisfeitas com os procedimentos transexualizadores realizados; apenas $9 \%$ conseguiram mudar o nome em pelo menos um documento; $71 \%$ relataram ter sido tratadas pelo nome social em mais de 4 lugares frequentados, 28\% declararam estar em tratamento para HIV e 22\% já ficaram presas por mais de 24 horas pelo menos uma vez. Noventa e quatro por cento (94\%) sofreram algum tipo de violência na vida e $57 \%$ relataram chantagem, extorsão ou violência policial.

O escore de bem-estar psicológico médio observado foi 63,2 (IC95\%: 61.8-64.6) (Tabela 1).

$\mathrm{Na}$ análise bivariada, não ter endereço fixo, ter menor escolaridade, estar insatisfeita com as relações pessoais, com o suporte de amigos ou com os procedimentos transexualizadores, ter sofrido algum tipo de violência e já ter ficado presa foram associados ao menor bem-estar psicológico. Por outro lado, ter maior renda mensal, ter algum trabalho e ser tratada pelo nome social nos lugares que frequenta foram associados ao maior bem-estar psicológico (Tabela 2). Após o ajuste das variáveis, mantiveram-se associadas ao menor bem-estar psicológico: não ter endereço fixo; ter menor escolaridade; estar insatisfeita com as relações pessoais, suporte de amigos ou procedimentos transexualizadores realizados; e ter sofrido violência verbal ou sexual. Somente "ter algum trabalho" manteve associação positiva ao bem-estar psicológico. As variáveis que apresentaram p > 0,05 e que não ajustaram as demais variáveis foram retiradas do modelo final (Tabela 2). 
Tabela 2

Análises bivariada e múltipla dos fatores associados ao bem-estar psicológico. São Paulo, Brasil, 2015.

\begin{tabular}{|c|c|c|c|c|c|}
\hline & \multirow{2}{*}{$\begin{array}{l}\text { WHOQOL-BREF } \\
\text { Média (DP) }\end{array}$} & \multicolumn{2}{|c|}{ Bivariada } & \multicolumn{2}{|c|}{ Modelo múltiplo } \\
\hline & & Coeficiente & Valor de $p$ & Coeficiente & Valor de $p$ \\
\hline \multicolumn{6}{|l|}{ Situação de moradia } \\
\hline Tem endereço fixo & $64,9(16,9)$ & - & - & - & - \\
\hline Sem endereço fixo & $54,7(19,3)$ & $-10,2$ & $<0,001$ & $-4,6$ & $<0,05$ \\
\hline \multicolumn{6}{|l|}{ Anos de escolaridade (anos) } \\
\hline$\geq 8$ & $65,1(17,5)$ & - & - & - & - \\
\hline$<8$ & $60,0(17,7)$ & $-5,1$ & $<0.05$ & $-4,1$ & $<0,05$ \\
\hline \multicolumn{6}{|l|}{ Renda (salários mínimos) } \\
\hline Até meio & $59,4(19,8)$ & - & - & & \\
\hline Até 1 & $59,9(18,5)$ & 0,5 & 0,855 & & \\
\hline Até 2 & $64,6(16,0)$ & 5,2 & $<0.05$ & & \\
\hline Mais de 2 & $65,0(17,4)$ & 5,6 & $<0.05$ & & \\
\hline \multicolumn{6}{|l|}{ Cor da pele } \\
\hline Branca, amarela, indígena & $62,6(17,9)$ & - & - & & \\
\hline Preta ou parda & $63,7(17,6)$ & 1,1 & 0,475 & & \\
\hline \multicolumn{6}{|l|}{ Trabalha } \\
\hline Não & $56,2(18,8)$ & - & - & - & - \\
\hline Sim, profissional do sexo & $63,7(16,8)$ & 7,5 & $<0,001$ & 3,9 & $<0,05$ \\
\hline Sim, outras atividades & $67,8(16,5)$ & 11,6 & $<0,001$ & 5,9 & $<0,05$ \\
\hline \multicolumn{6}{|l|}{ Está insatisfeita com as relações pessoais } \\
\hline Não & $66,1(16,0)$ & - & - & - & - \\
\hline Sim & $50,0(19,1)$ & $-16,1$ & $<0,001$ & $-10,7$ & $<0,001$ \\
\hline \multicolumn{6}{|l|}{ Está insatisfeita com o suporte de amigos } \\
\hline Não & $66,3(16,1)$ & - & - & - & - \\
\hline Sim & $53,5(19,0)$ & $-12,8$ & $<0,001$ & $-7,4$ & $<0,001$ \\
\hline \multicolumn{6}{|l|}{ Relação com a família } \\
\hline Nenhuma & $60,8(21,6)$ & - & - & & \\
\hline Conflituosa/Muito ruim & $55,3(19,1)$ & $-5,5$ & 0,064 & & \\
\hline Aceitável/Boa & $65,4(16,3)$ & 4,6 & 0,075 & & \\
\hline \multicolumn{6}{|l|}{ Tem alguma religião } \\
\hline Não & $61,7(16,8)$ & - & - & & \\
\hline Sim & $63,8(18,1)$ & 2,0 & 0,209 & & \\
\hline \multicolumn{6}{|l|}{ Tem parceiro fixo } \\
\hline Sim & $64,5(16,2)$ & - & - & & \\
\hline Não & $62,6(18,3)$ & $-1,9$ & 0,22 & & \\
\hline \multicolumn{6}{|l|}{ Está insatisfeita com os procedimentos realizados } \\
\hline Não & $65,1(16,9)$ & - & - & - & - \\
\hline Sim (pouco satisfeita/insatisfeita) & $59,2(17,4)$ & $-5,9$ & $<0,001$ & $-3,3$ & $<0,05$ \\
\hline Não fez nenhum procedimento & $62,5(21,7)$ & $-2,6$ & 0,301 & $-1,0$ & 0,663 \\
\hline \multicolumn{6}{|l|}{$\begin{array}{l}\text { Tentou e conseguiu mudar o nome em pelo menos um } \\
\text { documento }\end{array}$} \\
\hline $\begin{array}{l}\text { Não (ainda não tentou/tentou e não conseguiu/não tem } \\
\text { documento/está tentando) }\end{array}$ & $63,0(17,7)$ & - & - & & \\
\hline Não pretende mudar em nenhum dos documentos & $51,3(31,8)$ & $-11,7$ & 0,063 & & \\
\hline Sim & $67,9(14,4)$ & 5,0 & 0,063 & & \\
\hline \multicolumn{6}{|l|}{ Número de locais em que é tratada pelo nome social } \\
\hline Até 4 & $57,8(19,7)$ & - & - & & \\
\hline $44-6$ & $63,9(16,2)$ & 6,1 & $<0.05$ & & \\
\hline 7 ou mais & $67,1(16,3)$ & 9,4 & $<0,001$ & & \\
\hline
\end{tabular}

(continua) 


\begin{tabular}{|c|c|c|c|c|c|}
\hline & \multirow{2}{*}{$\begin{array}{c}\text { WHOQOL-BREF } \\
\text { Média (DP) }\end{array}$} & \multicolumn{2}{|c|}{ Bivariada } & \multicolumn{2}{|c|}{ Modelo múltiplo } \\
\hline & & Coeficiente & Valor de $p$ & Coeficiente & Valor de $p$ \\
\hline \multicolumn{6}{|l|}{ Violência } \\
\hline Não sofreu violência & $72,4(14,8)$ & - & - & - & - \\
\hline \multicolumn{6}{|l|}{ Humilhação } \\
\hline Agressão física & $63,5(20,5)$ & $-8,9$ & $<0.05$ & $-5,1$ & 0,133 \\
\hline Violência sexual & $62,5(16,5)$ & $-9,9$ & $<0.05$ & $-7,8$ & $<0.05$ \\
\hline Chantagem/Extorsão ou violência policial & $62,3(17,7)$ & $-10,1$ & $<0.05$ & $-5,2$ & 0,08 \\
\hline \multicolumn{6}{|l|}{ Já foi presa } \\
\hline Não & $64,0(17,2)$ & - & - & & \\
\hline Sim & $59,7(18,4)$ & $-4,4$ & $<0.05$ & & \\
\hline \multicolumn{6}{|l|}{ Faz tratamento para HIV } \\
\hline Não & $63,8(18,0)$ & - & - & & \\
\hline Sim & $61,7(16,9)$ & $-2,0$ & 0,213 & & \\
\hline
\end{tabular}

DP: desvio padrão; WHOQOL-BREF: World Health Organization Quality of Life.

\section{Discussão}

De maneira geral, melhores condições de moradia, ter trabalho e maior escolaridade são preditores de maior bem-estar psicológico entre travestis e mulheres transexuais. Tais achados são consistentes com estudos realizados fora do Brasil 44,45. Do ponto de vista das relações sociais, não estar satisfeita com as relações pessoais e com o suporte de amigos impacta negativamente o bem-estar psicológico 44,46. No nosso estudo, a perda de significância da variável sobre o relacionamento com a família no modelo múltiplo da análise de variância e sua associação com a variável insatisfação com o suporte de amigos sugerem que estas duas dimensões possam estar relacionadas. Na medida em que pessoas transgênero são expulsas de casa ou são obrigadas a deixar o convívio familiar, elas encontram nas redes de amigos, organizações comunitárias ou não governamentais o acolhimento de sua identidade/ expressão de gênero, e podem estabelecer e fortalecer relações de suporte e confiança 39.

Os escores médios do domínio psicológico encontrados entre travestis e mulheres transexuais foram ligeiramente inferiores aos observados entre mulheres transexuais em São Francisco, Estados Unidos 47. Em contrapartida, o escore do presente trabalho foi superior ao observado entre mulheres e homens transexuais em Barcelona, Espanha 45, sugerindo ser interessante identificar respostas contextuais que possam impactar tal domínio.

A insatisfação com os procedimentos transexualizadores realizados esteve associada ao menor bem-estar psicológico. Estudos mostram que os procedimentos transexualizadores têm impacto positivo na saúde mental das mulheres transgênero 48,49 . No entanto, os resultados positivos observados no bem-estar psicológico das mulheres transgênero não são diretos, mas mediados: as transformações físicas diminuem o estresse associado a não ser reconhecida como mulher e, consequentemente, diminui a chance de constrangimentos e violências 49,50. É possível também que as expectativas sobre os efeitos da intervenção não tenham sido completamente atendidas, considerando, por exemplo, que a transformação física obtida com a hormonoterapia varia de acordo com a idade de início do procedimento e com as características individuais. Na busca pela transformação do corpo, "desejo, sonho, necessidade e sobrevivência se misturam" 50 (p. 2521). Nesse sentido, a satisfação com o resultado é um indicador importante do impacto do procedimento no bem-estar psicológico. Não foi possível analisar o acesso ao processo transexualizador pelo SUS, mas a literatura sugere que o reconhecimento da identidade de gênero e a perspectiva de ter apoio para transformar o corpo podem resultar em maior bem-estar psicológico 50 . Não foi possível explorar se a insatisfação relatada estava associada à falta de acesso a serviços e profissionais adequados. 
Além das questões relativas às transformações corporais, o contexto de vida de travestis e mulheres transexuais apresenta contornos particulares sobre as formas de impacto da violência urbana e de gênero, bem como as estratégias de enfrentamento. Uma em cada três travestis ou mulheres transexuais já viveu pelo menos uma forma de violência em decorrência de sua expressão e identidade de gênero. A maioria já foi vítima de violência policial ou de chantagem e extorsões. No que pese não termos realizado tal análise, tais experiências são frequentemente associadas ao contexto de prostituição e/ou de envolvimento com o uso e tráfico de drogas 51.

Em nossa amostra, comparativamente às pessoas que se autoidentificam como mulheres transexuais, as que se identificam como travestis apresentam marcadores de maior vulnerabilidade social, tais como menor escolaridade, cor da pele preta ou parda, piores condições de moradia e maior envolvimento com prostituição (comparação não exibida no presente trabalho). No modelo múltiplo, a variável identidade de gênero não foi significativa na presença das variáveis sociodemográficas (dados não mostrados). Possivelmente o que separa os dois grupos em relação ao bem-estar é a maior vulnerabilidade social experimentada pelas travestis. É importante reconhecer que tais categorias são produções culturais, que podem e são modificadas ao longo da história 52. Se historicamente no Brasil a categoria travesti apareceu inicialmente associada à prostituição, hoje é possível observar, com frequência, nos espaços públicos, travesti ser a categoria de identidade de gênero referida entre ativistas, especialmente entre as de maior escolaridade 7.

Particularmente no que diz respeito à violência verbal exclusiva, estudos observaram que esta é recorrente entre a população estudada por meio de xingamentos, ofensas e episódios de discriminação, inclusive em serviços de saúde quando vão em busca de ajuda após uma violência física e/ou sexual ${ }^{39}$. É possível que o mecanismo para a associação entre a violência verbal exclusiva com o pior bem-estar esteja relacionado à recorrência deste tipo de violência em vários ambientes frequentados por travestis e mulheres transexuais, inclusive quando em busca de ajuda frente à vitimização por outros tipos de violência 40 .

As questões relacionadas à violência, seja como vítima ou perpetrador em suas relações macro e micro sociais, são de natureza complexa. Bourdieu define violência simbólica como um tipo invisível de agressão que acontece por meio da dominação imposta pelos mais privilegiados àqueles que pertencem a um grupo socialmente estigmatizado. Tais atos se desenvolvem no âmbito de relações de poder que resultam na opressão e vitimização das minorias no ambiente familiar, das instituições e das condições políticas e econômicas. Isso leva a uma naturalização inconsciente da posição de vítima e à consequente aceitação de seu papel social restrito à sua condição econômica e minoritária 53. Esse tipo de agressão está diluído em diversos espaços de circulação das travestis e pode constituir uma das explicações para a ausência de diferença nos tipos de violência, apesar de viverem em condições mais suscetíveis à sua ocorrência 54 .

Reconhecer a atividade exercida como trabalho, independentemente do tipo de ocupação, tem impacto positivo sobre o bem-estar psicológico entre travestis e mulheres transexuais. Especificamente em relação ao trabalho sexual, embora o envolvimento com a atividade de prostituição constitua um fator de risco para infecção por HIV, uso prejudicial de drogas, transtornos mentais 55 , assim como maior susceptibilidade a situações de violência física, sexual, e prisões arbitrárias 56 , dentre outros, o reconhecimento da prostituição como identidade profissional apresenta características particulares. Independentemente da identidade ou expressão de gênero, a pessoa que significa a prostituição como trabalho reconhece a prática de sexo comercial como algo legítimo e, a exemplo das experiências do ativismo como, por exemplo, na criação da Rede Brasileira de Prostitutas, a prostituição assume um espaço de reivindicação de protagonismo e defesa de direitos humanos.

O presente estudo apresenta limitações, dentre as quais o fato de a seleção amostral não ser probabilística e ter sido feita em serviços de saúde e de assistência social, inclusive nos serviços de atenção à saúde das pessoas transexuais, o que pode ter resultado em superestimação do desfecho. Entretanto, com populações consideradas escondidas ou de difícil acesso, a amostragem do tipo bola de neve é extensivamente utilizada por facilitar a identificação e o contato, como neste trabalho.

O desenho de estudo transversal não permite em geral identificar a temporalidade dos fatos, o que dificulta o estabelecimento de relações causais. No entanto, foi apropriado para analisarmos os fatores associados ao nosso desfecho, que independentemente da relação causal, podem ser indicativos para a implantação de políticas públicas a esta população. 
Destacamos que, apesar das limitações relativas ao desenho do estudo e seleção amostral, os escores e fatores associados ao bem-estar observados foram similares aos de trabalhos nacionais e internacionais que utilizaram outras abordagens metodológicas mais robustas, além de ter permitido uma amostra de tamanho suficiente para as devidas estimativas.

No nosso estudo, condições de desigualdade social e de exposição à violência simbólica e sexual estão associadas a um pior bem-estar psicológico de travestis e mulheres transexuais. Por outro lado, apesar da alta prevalência, a ausência de efeito da violência física e de chantagem, extorsão ou violência policial no bem-estar psicológico pode ser lida como a expressão de resiliência, resultado de um aprendizado de resistência e enfrentamento a estas condições do ponto de vista psicossocial. Mesmo que não plenamente, conseguir realizar transformações corporais desejadas e o respeito ao nome social materializam o reconhecimento da legitimidade de uma identidade ou expressão de se "poder ser quem se é de verdade”.

São necessárias investigações que possam aferir, no plano populacional, os efeitos dos vários tipos de violência em contextos-chave, assim como as repercussões de experiências de viver na rua, institucionalização e encarceramento, sobretudo na saúde mental. Do ponto de vista das políticas de saúde, torna-se central conceber o cuidado integral de saúde para pessoas travestis e transexuais com base nas suas necessidades e demandas. Considerar o espectro das experiências da transgeneridade, as especificidades das questões relativas às mudanças corporais no conjunto de dimensões referentes à qualidade de vida, na perspectiva de que o direito à livre expressão do gênero é um direito humano.

\section{Colaboradores}

E. M. Zucchi, C. R. S. Barros, B. R. L. Redoschi, L. F. A. Deus e M. A. S. M. Veras contribuíram na concepção do projeto, análise e interpretação dos dados; redação do artigo e revisão crítica relevante do conteúdo intelectual; aprovação final da versão a ser publicada; e são responsáveis por todos os aspectos do trabalho na garantia da exatidão e integridade de qualquer parte da obra.

\section{Informações adicionais}

ORCID: Eliana Miura Zucchi (0000-0001-62341490); Claudia Renata dos Santos Barros (00000002-1582-2010); Bruna Robba Lara Redoschi (0000-0002-0319-3835); Luiz Fabio Alves de Deus (0000-0002-9868-3182); Maria Amélia de Sousa Mascena Veras (0000-0002-1159-5762).

\section{Agradecimentos}

O projeto foi financiado pela Fundação de Amparo à Pesquisa do Estado de São Paulo (FAPESP) - processo 2013/22366-7.

\section{Referências}

1. Reisner SL, Poteat T, Keatley JA, Cabral M, Mothopeng T, Dunham E, et al. Global health burden and needs of transgender populations: a review. Lancet 2016; 388:412-36.

2. Thomas R, Pega F, Khosla R, Verster A, Hana T, Sayc L. Ensuring an inclusive global health agenda for transgender people. Bull World Health Organ 2017; 95:154-6.

3. Popadiuk GS, Oliveira DC, Signorelli MC. A Política Nacional de Saúde Integral de Lésbicas, Gays, Bissexuais e Transgêneros (LGBT) e o acesso ao processo transexualizador no Sistema Único de Saúde (SUS): avanços e desafios. Ciênc Saúde Coletiva 2017; 22:1509-20.

4. Schuster MA, Reisner SL, Onorato SE. Beyond bathrooms - meeting the health needs of transgender people. N Engl J Med 2016; 375:101-3.

5. Stryker S. Historia de lo trans: las raíces de la revolución de hoy. Madrid: Continta me Tienes; 2017.

6. Carvalho M, Carrara S. Em direito a um futuro trans?: contribuição para a história do movimento de travestis e transexuais no Brasil. Sex Salud Soc (Rio J.) 2013; (14):319-51.

7. Barbosa BC. "Doidas e putas": usos das categorias travesti e transexual. Sex Salud Soc (Rio J.) 2013; (14):352-79. 
8. Hoy-Ellis CP, Fredriksen-Goldsen KI. Depression among transgender older adults: general and minority stress. Am J Community Psychol 2017; 59:295-305.

9. Hwahng SJ, Nuttbrock L. Adolescent gender-related abuse, androphilia, and HIV risk among transfeminine people of color in New York City. J Homosex 2014; 61:691-713.

10. Irwin JA, Coleman JD, Fisher CM, Marasco VM. Correlates of suicide ideation among LGBT Nebraskans. J Homosex 2014; 61:1172-91.

11. Leinung M, Urizar M, Patel N, Sood S. Endocrine Treatment of Transsexual Persons: Extensive Personal Experience. Endocr Pract 2013; 19:644-50.

12. Nuttbrock L, Bockting W, Rosenblum A Hwahng S, Mason M, Macri M, et al. Gender abuse, depressive symptoms, and HIV and other sexually transmitted infections among male-to-female transgender persons: a threeyear prospective study. Am J Public Health 2013; 103:300-7.

13. Nuttbrock L, Bockting W, Rosenblum A, Hwahng S, Mason M, Macri M, et al. Gender abuse, depressive symptoms, and substance use among transgender women: a 3-year prospective study. Am J Public Health 2014; 104:2199-206.

14. Bockting WO, Miner MH, Swinburne Romine RE, Hamilton A, Coleman E. Stigma, mental health, and resilience in an online sample of the US transgender population. Am J Public Health 2013; 103:943-51.

15. Steptoe A, Deaton A, Stone AA. Psychological wellbeing, health and ageing. Lancet 2015; 385:640-8.

16. Machado WL, Bandeira DR. Bem-estar psicológico: definição, avaliação e principais correlatos. Estud Psicol (Campinas) 2012; 29:58795.

17. Edmondson OJ, MacLeod AK. Psychological well-being and anticipated positive personal events: their relationship to depression. Clin Psychol Psychother 2015; 22:418-25.

18. Nierenberg AA, Rapaport MH, Schettler PJ, Howland RH, Smith JA, Edwards D, et al. Deficits in psychological well-being and qualityof-life in minor depression: implications for DSM-V. CNS Neurosci Ther 2010; 16:208-16.

19. Davey A, Bouman WP, Arcelus J, Meyer C. Social support and psychological well-being in gender dysphoria: a comparison of patients with matched controls. J Sex Med 2014; 11:2976-85.

20. Giuliani C, Tagliabue S, Regalia C. Psychological well-being, multiple identities, and discrimination among first and second generation immigrant Muslims. Eur J Psychol 2018; 14:66-87.

21. Warren JC, Smalley KB, Barefoot N. Psychological well-being among transgender and genderqueer individuals. Int J Transgend 2016; 17:114-23.

22. Parker R, Aggleton P. HIV and AIDS-related stigma and discrimination: a conceptual framework and implications for action. Soc Sci Med 2003; 57:13-24.
23. Maluwa M, Aggleton P, Parker R. HIV and AIDS-related stigma, discrimination, and human rights. Health Hum Rights 2002; 6:1-18.

24. Clements-Nolle K, Marx R, Katz M. Attempted suicide among transgender persons: the influence of gender-based discrimination and victimization. J Homosex 2006; 51:53-69.

25. Gamarel KE, Reisner SL, Laurenceau J-P, Nemoto T, Operario D. Gender minority stress, mental health, and relationship quality: a dyadic investigation of transgender women and their cisgender male partners. J Fam Psychol 2014; 28:437-47.

26. Nuttbrock L, Bockting W, Rosenblum A, Hwahng S, Mason M, Macri M, et al. Gender abuse and major depression among transgender women: a prospective study of vulnerability and resilience. Am J Public Health 2014; 104:2191-8.

27. Keuroghlian AS, Reisner SL, White JM, Weiss RD. Substance use and treatment of substance use disorders in a community sample of transgender adults. Drug Alcohol Depend 2015; 152:139-46.

28. Yang X, Zhao L, Wang L, Hao C, Gu Y, Song W, et al. Quality of life of transgender women from china and associated factors: a cross-sectional study. J Sex Med 2016; 13:977-87.

29. Blosnich JR, Marsiglio MC, Gao S, Gordon AJ, Shipherd JC, Kauth M, et al. Mental health of transgender veterans in US States with and without discrimination and hate crime legal protection. Am J Public Health 2016; 106:53440.

30. Szymanski DM, Gupta A. Examining the relationship between multiple internalized oppressions and African American lesbian, gay, bisexual, and questioning persons' self-esteem and psychological distress. J Couns Psychol 2009; 56:110-8.

31. Nemoto T, Bödeker B, Iwamoto M. Social support, exposure to violence and transphobia, and correlates of depression among male-to-female transgender women with a history of sex work. Am J Public Health 2011; 101:1980-8.

32. Brown GR, Jones KT. Health correlates of criminal justice involvement in 4,793 transgender veterans. LGBT Health 2015; 2:297305.

33. Routh D, Abess G, Makin D, Stohr MK, Hemmens C, Yoo J. Transgender inmates in prisons. Int J Offender Ther Comp Criminol 2017; 61:645-66.

34. Amaral MS, Silva TC, Cruz KO, Toneli MJF. "Do travestismo às travestilidades": uma revisão do discurso acadêmico no Brasil entre 2001-2010. Psicol Soc 2014; 26:301-11.

35. Carrara S, Vianna ARB. Tá lá o corpo estendido no chão: a violência letal contra travestis no Município do Rio de Janeiro. Lethal violence against travestis in Rio de Janeiro city. Physis (Rio J.) 2006; 16:233-49.

36. Aguinsky BG, Ferreira GG, Rodrigues MC. Travestis e segurança pública: as performances de gênero como experiências com o sistema e a política de segurança no Rio Grande do Sul. Textos Contextos (Porto Alegre) 2013; 12:47-54. 
37. Sousa PJ, Ferreira LOC, Sá JB. Estudo descritivo da homofobia e vulnerabilidade ao HIV/ Aids das travestis da Região Metropolitana do Recife, Brasil TT. Ciênc Saúde Coletiva 2013; 18:2239-51.

38. Trasngender Europe; Trans Respect Versus Transphobia Worldwide. TMM Update. Trans day of remembrance 2017. https://transre spect.org/wp-content/uploads/2017/11/ TvT_TMM_TDoR2017_Tables_EN.pdf (acessado em 16/Mar/2018).

39. Souza MHT, Malvasi P, Signorelli MC, Pereira PPG, Souza MHT, Malvasi P, et al. Violência e sofrimento social no itinerário de travestis de Santa Maria, Rio Grande do Sul, Brasil. Cad Saúde Pública 2015; 31:767-76.

40. Silva GWS, Souza EFL, Sena RCF, Moura IBL, Sobreira MVS, Miranda FAN. Situações de violência contra travestis e transexuais em um município do nordeste brasileiro. Rev Gaúch Enferm 2016; 37:e56407.

41. Mello L, Avelar RB, Brito W. Políticas públicas de segurança para a população LGBT no Brasil. Revista Estudos Feministas 2014; 22:297320.

42. Taquette SR. Conduta ética no atendimento à saúde de adolescentes. Adolescência \& Saúde 2010; 7:6-11.

43. Silva R, Bezerra W, Queiroz S. Os impactos das identidades transgênero na sociabilidade de travestis e mulheres transexuais. Rev Ter Ocup 2015; 26:364-72.

44. Stanton MC, Ali S, Chaudhuri S. Individual, social and community-level predictors of wellbeing in a US sample of transgender and gender non-conforming individuals. Cult Health Sex 2017; 19:32-49.

45. Gómez-Gil E, Zubiaurre-Elorza L, Antonio IE, Guillamon A, Salamero M. Determinants of quality of life in Spanish transsexuals attending a gender unit before genital sex reassignment surgery. Qual Life Res 2014; 23:669-76.

46. Koenig HG, Zaben FA, Khalifa DA. Religion, spirituality and mental health in the West and the Middle East. Asian J Psychiatr 2012; 5:180-2.
47. Thompson HM, Reisner SL, VanKim N, Raymond HF. Quality-of-life measurement: assessing the WHOQOL-BREF scale in a sample of high-HIV-risk transgender women in San Francisco, California. Int J Transgend 2015; 16:36-48.

48. Weyers S, Elaut E, De Sutter P, Gerris J, T'Sjoen G, Heylens G, et al. Long-term assessment of the physical, mental, and sexual health among transsexual women. J Sex Med 2009; 6:752-60.

49. Colizzi M, Costa R, Pace V, Todarello O. Hormonal treatment reduces psychobiological distress in gender identity disorder, independently of the attachment style. J Sex Med 2013; 10:3049-58

50. Rocon PC, Rodrigues A, Zamboni J, Pedrini MD. Dificuldades vividas por pessoas trans no acesso ao Sistema Único de Saúde. Ciênc Saúde Coletiva 2016; 21:2517-26.

51. Odinokova V, Rusakova M, Urada LA, Silverman JG, Raj A. Police sexual coercion and its association with risky sex work and substance use behaviors among female sex workers in St. Petersburg and Orenburg, Russia. Int J Drug Policy 2014; 25:96-104.

52. Arán M, Murta D. Do diagnóstico de transtorno de identidade de gênero às redescrições da experiência da transexualidade: uma reflexão sobre gênero, tecnologia e saúde. Physis (Rio J.) 2009; 19:15-41.

53. Minayo MCS. Violência social sob a perspectiva da saúde pública. Cad Saúde Pública 1994; 10 Suppl 1:S7-18

54. Carrieri AP, Souza EM, Aguiar ARC. Trabalho, violência e sexualidade: estudo de lésbicas, travestis e transexuais. Revista de Administração Contemporânea 2014; 18:78-95.

55. Logie $\mathrm{CH}$, Wang $\mathrm{Y}$, Lacombe-Duncan A, Jones $\mathrm{N}$, Ahmed U, Levermore K, et al. Factors associated with sex work involvement among transgender women in Jamaica: a cross-sectional study. J Int AIDS Soc 2017; 20:21422.

56. Decker MR, Crago A-L, Chu SKH, Sherman SG, Seshu MS, Buthelezi K, et al. Human rights violations against sex workers: burden and effect on HIV. Lancet 2015; 385:186-99. 


\section{Abstract}

This article sought to estimate factors associated with the psychological well-being of transvestites and trans women. It is a cross-sectional study with 602 transvestites and trans individuals in seven cities in the state of São Paulo, Brazil from 2014 to 2015. We carried out a sample selection through a consecutive approach and using the snowball technique. The dependent variable was psychological well-being (WHOQOL-BREF) and the independent variables were: sociodemographic characteristics, body modifications, health conditions, violence and incarceration. We used a multiple variance analysis to identify associated factors. Most were black or brown and were aged between 25 and 39 years, had up to complete secondary education, individual income of up to two times the minimum wage and worked, and $42.3 \%$ were sex workers. Around one-quarter had been incarcerated. Around one-quarter were in treatment for HIV. Mean psychological well-being score was 63.2 (95\%CI: 61.8-64.6). In the multiple analysis, the factors associated with lower psychological well-being were: not having a fixed address, having lower educational levels, being dissatisfied with personal relationships, friend support or the gender-affirming procedures they had undergone and having suffered verbal or sexual violence. While worse living conditions and exposure to violence harm the psychological well-being of transvestites and trans women, the possibility of undergoing desired body transformations and having their social name respected interfere positively in their evaluations of their lives.

Transvestism; Transgender Persons; Mental Health

\section{Resumen}

El objetivo fue estimar factores asociados al bienestar psicológico de travestis y mujeres transexuales. Se trata de un estudio transversal con 602 travestis y personas transexuales en siete municipios del estado de São Paulo, Brasil, entre 2014 y 2015. Se realizó una selección de muestras con un enfoque consecutivo y técnica bola de nieve. La variable dependiente fue bienestar sicológico (WHOQOL$B R E F)$ y las independientes fueron: características sociodemográficas, modificaciones corporales, condiciones de salud, violencia y encarcelamiento. Se usó un análisis multivariante de variancia para identificar los factores asociados. La mayoría tenía el color de piel negro o eran mulatos, y entre 25 y 39 años de edad, con hasta la enseñanza media completa, renta individual de hasta dos salarios minimos y trabajaba, siendo un 42,3\% profesionales del sexo. Cerca de un cuarto ya estuvo presa. También cerca de un cuarto estaba con tratamiento para el VIH. Las puntuaciones medias de bienestar sicológico fue 63,2 (IC95\%: 61,8-64,6). En el análisis múltiple, estuvieron asociados a un menor bienestar sicológico: no tener dirección fija, tener menor escolaridad, estar insatisfecha con las relaciones personales, apoyo de amigos o procedimientos transexualizadores realizados, y haber sufrido violencia verbal o sexual. Mientras que unas peores condiciones de vida y de exposición a la violencia perjudican el bienestar sicológico de travestis y mujeres transexuales, la posibilidad de realizar las transformaciones corporales deseadas y el respeto al nombre social interfieren positivamente en la evaluación que hacen de sus vidas.

Travestismo; Personas Transgénero; Salud Mental
Recebido em 29/Mar/2018

Versão final reapresentada em 21/Nov/2018

Aprovado em 04/Dez/2018 